\title{
Characterisation of handling and service surface damage on Nickel alloys caused by low velocity impacts of blunt hard objects
}

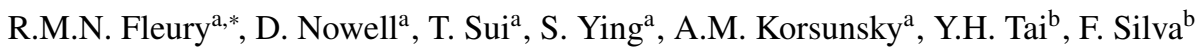 \\ ${ }^{a}$ Department of Engineering Science, University of Oxford, Parks Road, OX1 3PJ, Oxford, UK \\ ${ }^{b}$ Rolls-Royce plc, P.O. Box 31, DE24 8BJ, Derby, UK
}

\begin{abstract}
This paper presents a characterisation of surface damage, more specifically dents, caused by low velocity impacts of blunt objects on RR1000 Nickel superalloys. These are representative of damage that may occur during handling and service of components during manufacturing or maintenance. The characterisation of dents produced in laboratory tests is carried out both in terms of their geometry and the residual stresses the damage. A finite element model is presented and the results are validated in terms of dent geometry produced for different impact velocities. The stress distribution predicted by the numerical model is also compared with experimentally measured stresses via X-ray diffraction for validation of the model. The residual stresses obtained from the finite element (FE) model and their implications to fatigue and crack propagation lives are also discussed here.
\end{abstract}

Keywords: surface damage; dents; impact mechanics; residual stresses; Nickel superalloys

\section{Introduction}

Low velocity impacts by hard metallic objects may cause surface damage on components of aircraft gas turbines. Damage caused by hard objects in this velocity range (i.e. impact velocities of $1-15 \mathrm{~m} / \mathrm{s}$ ) is representative of events that may occur during manufacturing or repair of engines, when tools or parts might be dropped and small dents or scratches may occur. Such events are sometimes referred to as handling and service object damage. The effects of handling and service damage on the fatigue life of components is frequently estimated by empirical methods or ignored altogether. The work of Gourdin et al. $(2014,2016)$ on crack propagation near surface anomalies is one of the few references to handling damage in the literature. Currently, a crack propagation analysis of components with small scratches and dents is frequently made by assuming the damage geometry to be an initial crack of same length and depth. This approach is generally very conservative, since it takes into account neither the residual stress field generated near the damage nor the time that it takes for the crack to initiate from the stress concentrator. In improving the efficiency and safety of aircraft engines, and to comply with changing safety requirements, manufacturers need to improve their understanding of the influence of surface damage on the fatigue life of components. In order to accurately predict the effect of such surface damage, it is first necessary to predict the geometry of the damage geometry caused by a given impact together with the residual stress field in the vicinity.

Using the potential drop technique, Gourdin et al. (2016) quantified the difference in crack initiation and propagation time between scratches and dents of similar geometry. Experiments were undertaken in the presence and absence of residual stresses; the latter achieved by a thermal treatment of the specimens in order to relax the residual stresses. Their study has shown that compressive residual stresses significantly slow down crack growth and therefore extend fatigue life. A similar study was carried out by Doremus et al. (2015) using Inconel 718 specimens. Here, it was shown that the initiation life in dented specimens reduces once the residual stresses are removed, whereas scratches show the opposite effect. It was also demonstrated that crack initiation in samples with surface damage is influenced

\footnotetext{
${ }^{*}$ Corresponding author.

Email address: rodolfo.fleury@eng.ox.ac.uk (R.M.N. Fleury)
} 
by a combination of three factors: stress concentration due to the geometry of the notch, the residual stress field created, and the micro-structural changes by the deformation. These are the same three factors observed by Mall et al. (2001) when investigating the impact of foreign object damage (FOD) on high cycle fatigue in Titanium alloys. Doremus et al. (2015) used a static finite element (FE) model to explain, qualitatively, the shape of the crack front around the damage by comparing the residual stress distribution near the corner of the dent and under the root. Note that in both Gourdins and Doremus work the dent had sharp internal corners and hence significant edge effects were present.

More extensive work has been carried out on higher velocity impact events, such as foreign object damage (FOD). The impact of FOD on low and high cycle fatigue crack growth has been investigated by Ding et al. (2007a,b) and Peters et al. (2002). High speed impact events generally cause severe structural damage and complex mechanisms of failure often need to be modelled (Nowell et al., 2003). Despite the significantly lower impact energy in handling and service damage, some analogies can be drawn with FOD problems. As shown previously, both have a competing influence on the crack growth of: i) the stress concentration due to the presence of the geometrical notch; and ii) the residual stress field, particularly the compressive zone. Duó et al. (2007) have used laboratory and synchrotron X-ray diffraction (XRD) to measure the residual stresses caused by FOD. Although good qualitative agreement between measured residual stresses and those modelled by finite element simulation, a discrepancy was found between the predicted and measured stress magnitudes. This discrepancy is mainly due to difficulty in modelling these high velocity impacts and the damage mechanisms caused by it. The residual stresses in the interior of specimens, caused by FOD were measured using synchrotron XRD Frankel et al. (2012). They also carried out a dynamic FE simulation using a Johnson-Cook strain rate dependent material model. Again, good qualitative agreement was obtained, but the magnitude of the stresses was over-predicted.

The problem of fatigue life prediction in the presence of handling and service damage involves some important unresolved questions in solid mechanics. Short crack propagation in the presence of a geometrical stress concentrator, the contribution of the residual stress field and the material damage/hardening near the root of the dent are some of the key questions that need to be answered. The current paper will extend the understanding in the literature by investigating the geometry of the damage and the residual stresses under it, particularly blunt dents in the RR1000 Nickel alloy due to low velocity impacts. A model for prediction of both the dent geometry and the residual stress field in its vicinity will be presented. First, a geometric characterisation of dents is carried out by experimentally creating dents on the specimens. The measured geometries are then used to validate a FE model of the impacts. The residual stress fields obtained from the numerical model is validated by comparing it with measurements using X-ray diffraction (XRD). The objective is to develop a model capable of predicting the two hey parameters highlighted above, namely the geometry of the dent, which act as a geometrical stress concentrator, and the residual stress distribution, which will influence both crack initiation and propagation.

\section{Experimental damage simulation}

\subsection{Experimental method}

Experimental damage simulation was carried out using a modified Hopkinson Bar gas gun at the University of Oxford (Fig. 1). The specimen was mounted perpendicular to the striker bar at the far end of the gun. The striker bar is a $10 \mathrm{~mm}$ diameter, $330 \mathrm{~mm}$ long, silver steel bar with an indenter mounted at the impact end. The indenter is made of hardened steel with a blunt profile generated by two perpendicular curved surfaces of different radii $\left(R_{1}=12.5 \mathrm{~mm}\right.$ and $R_{2}=1 \mathrm{~mm}$ ) as shown in Fig. 2. In order to damage a specimen, the solenoid valve is opened, allowing compressed air to exit from the reservoir and drive the piston down the barrel. The piston hits the striker bar and it, in turn, hits the specimen, producing the required damage. The impact velocity depends on the reservoir pressure and a high speed camera was used to obtain the pressure against velocity calibration.

Fatigue specimens with rectangular cross-section were used in the dent tests. These are standard fatigue specimens which may be tested after the damage is created on both flat faces. The central gauge length of the specimens has a cross-section of $5 \times 11 \mathrm{~mm}$ and a length of approximately $14 \mathrm{~mm}$. The specimens are made of the RR1000 Nickel superalloy with fine grain micro-structure, which has a composition as described in Table 1 (Bache et al., 2016; Mitchell et al., 2004). The surface of the specimens where the dents were created were milled and shot peened. However, the dents created were considerably deeper than the shot peened layer and the effect of the shot peening 


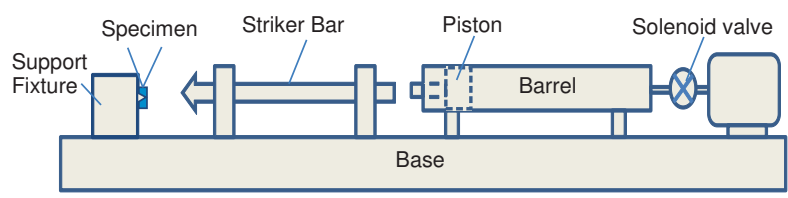

Figure 1: Schematic drawing of the Hopkinson bar gas gun adapted for low speed impacts.
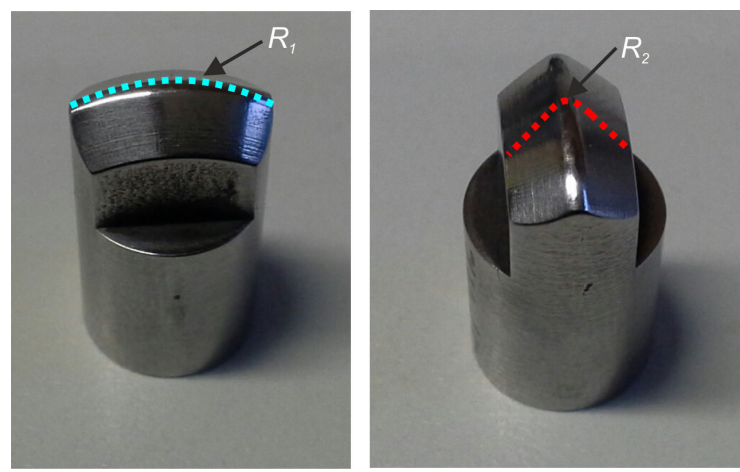

Figure 2: Hardened steel indenter tip profile and radii.

were neglected in the numerical analysis. Each dent was created in the centre of a flat face of the specimen. An example of the dent geometry on the face of a specimen is given in Fig. 3.

\begin{tabular}{ccccccccccc}
\multicolumn{1}{c}{ Table 1: RR1000 nominal composition (wt.\%, balance nickel). } \\
\hline Alloy & $\mathrm{Co}$ & $\mathrm{Cr}$ & $\mathrm{Mo}$ & $\mathrm{Ti}$ & $\mathrm{Al}$ & $\mathrm{Ta}$ & $\mathrm{Hf}$ & $\mathrm{Zr}$ & $\mathrm{C}$ & $\mathrm{B}$ \\
\hline RR1000 & 18.5 & 15 & 5 & 3.6 & 3 & 2 & 0.5 & 0.06 & 0.027 & 0.015 \\
\hline
\end{tabular}

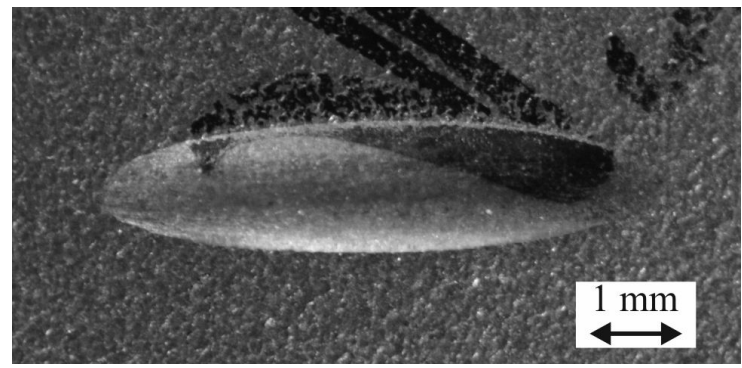

Figure 3: Example of a dent geometry on the flat face of the specimen.

The relationship between the impact velocity and the applied pressure was first carried out, since it is the applied pressure that is the input in the experimental tests, but it is the impact velocity of the striker bar which is used as an input in the FE model of the tests. A Photrom Fastcam high speed camera was positioned such that the indenter could be seen travelling forward and backwards, i.e. before and after the impact. The framing rate was set to 35,000 frames per second. A small mark on the striker bar next to the hardened steel indenter was used to track the position of the indenter at each frame. The displacement versus time plot of the indenter at each frame was then obtained by using the Imetrum Digital Image Correlation software. Both impact and rebound velocities were then calculated from the displacement history of the indenter. Knowledge of the impact velocity is important for subsequent FE modelling. The rebound velocity is also important since it gives a measure of the net energy dissipated during the impact. A linear correlation between the impact and rebound velocities was observed for the range of velocities studied (i.e. there was a constant coefficient of restitution of about 0.5). 


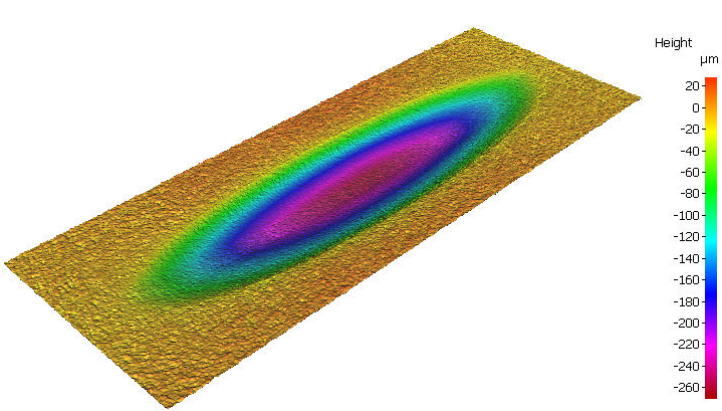

(a)

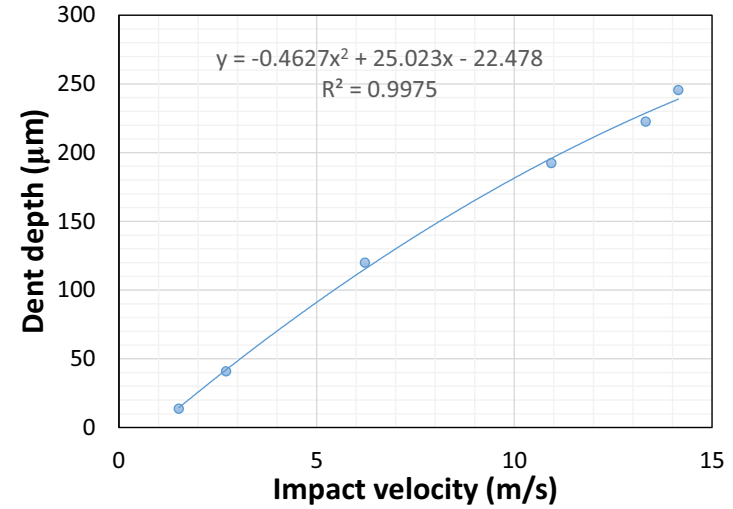

(b)

Figure 4: (a) 3D profile of the dent obtained with an Alicona InfiniteFocus microscope; (b) Dent depth as a function of the impact velocity.

\subsection{Dent geometry measurement}

The dent geometry created in each test was measured using an Alicona InfiniteFocus microscope. A representative 3D profile of a typical dent is presented in Fig. 4a. The dent observed on the specimen approximately matches the profile of the indenter. The length, width and depth of the dents were measured from the 3D Alicona profile and each was plotted as a function of the impact velocity. The correlation between dent depth and impact velocity is presented in Fig. 4b. A quadratic polynomial gives a reasonable fit between the experimental data and the impact velocity for the velocity range studied.

The list of tests carried out in this study is presented in Table 2. The applied pressure range is between 70-900 psi, resulting in dent depths between 50-250 $\mathrm{m}$ (1-10 thou). Dents of nominal depths of 1, 2 and 5 thou were easily generated. The 10 thou dents initially presented a problem of a double impact of the indenter (e.g. Test No. 5), where the striker bounced back from the piston and hit the specimen again. This phenomenon could readily be observed with the aid of the high speed camera. Surface damage on components caused by handling and service events generally do not have more than one impact on the same spot. Therefore, pair of roller clutch bearings were introduced to stop the striker bar after the first impact, eliminating the second impact. Once the issue of double impact was resolved, a few tests were carried out until the desired depth of about 10 thou was achieved (Test No. 8). A dent depth of approximately 10 thou $(246 \mu \mathrm{m})$ in a single strike was achieved with a pressure of 900 psi. Before the clutch was introduced, a similar depth was obtained with a pressure of only 500 psi only. This difference highlights the effect of the second impact on dent depth. Test numbers 1, 3, 6 and 8 were selected for subsequent finite element modelling and measurement of the residual stresses by XRD.

Table 2: Test list, dent geometry and impact velocity.

\begin{tabular}{cccccc}
\hline $\begin{array}{c}\text { Test } \\
\text { No. }\end{array}$ & $\begin{array}{c}\text { Pressure } \\
(\mathbf{p s i})\end{array}$ & $\begin{array}{c}\text { Length } \\
(\mathbf{m m})\end{array}$ & $\begin{array}{c}\text { Width } \\
(\mathbf{m m})\end{array}$ & $\begin{array}{c}\text { Depth } \\
(\boldsymbol{\mu} \mathbf{m})\end{array}$ & $\begin{array}{c}\text { Velocity } \\
(\mathbf{m} / \mathbf{s})\end{array}$ \\
\hline $\mathbf{1}$ & 220 & 4.54 & 1.05 & 127 & - \\
$\mathbf{2}$ & 70 & 1.45 & 0.34 & 14 & 1.52 \\
$\mathbf{3}$ & 100 & 2.58 & 0.65 & 41 & 2.71 \\
$\mathbf{4}$ & 220 & 4.50 & 1.05 & 120 & 6.22 \\
$\mathbf{5}$ & 500 & 6.10 & 1.41 & 252 & 11.55 \\
$\mathbf{6}$ & 600 & 5.85 & 1.46 & 193 & 10.94 \\
$\mathbf{7}$ & 800 & 6.36 & 1.59 & 223 & 13.32 \\
$\mathbf{8}$ & 900 & 6.53 & 1.66 & 246 & 14.15 \\
\hline
\end{tabular}




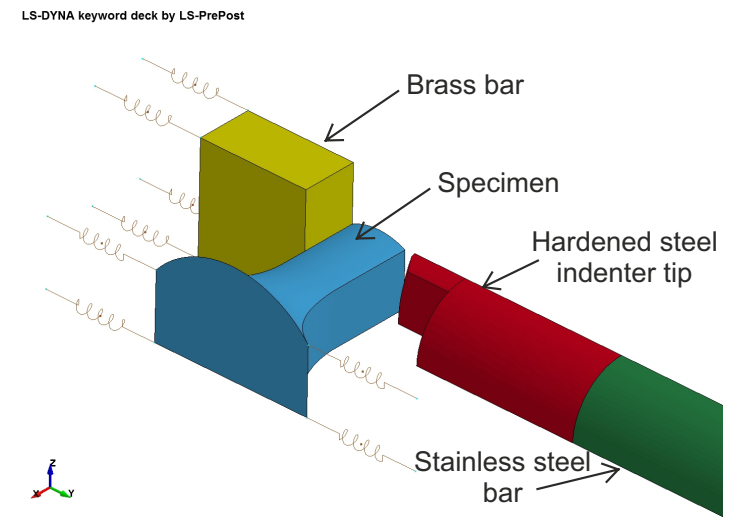

Figure 5: Model geometry and boundary conditions used in the LS-DYNA explicit FE code.

\section{Finite element model}

\subsection{Model geometry and boundary conditions}

Several authors have investigated damage due the impact of a hard indenter on metal alloys. Generally, these have been carried out for high impact velocity events, such as FOD impacts. Boyce et al. (2001) suggested that impact events of low impact velocities $(<200 \mathrm{~m} / \mathrm{s})$ can be modelled using a quasi-static approach. This corroborates the use of a static model by Doremus et al. (2015). Boyce et al. (2001) argued that by modelling an indentation with the same final dent depth, the stress field obtained is reasonably accurate and any discrepancy may be addressed by incorporating dynamic effects into the FE model. However, despite the low velocity range analysed in this paper, a dynamic analysis will be carried out since our goal is to be able to predict not only the residual stresses, but also the size of the dent created as a function of the impact velocity. Dynamic modelling of impact events have been implemented in the study of FOD (Duó et al., 2007; Frankel et al., 2012). These, however, are significantly more complex and evidence of strain-rate hardening, thermal softening, and material "tearing" effects have been reported (Nowell et al., 2003) Nevertheless, some of the techniques used to model FOD are used here to model handling and service damage.

The experimental impact tests were modelled using an explicit FE solver in LS-DYNA. The relevant components of the rig were included in the model, namely the indenter hardened steel tip, the stainless steel striker bar, the specimen, and the brass bar supporting the back of the specimen. This bar was placed behind the specimen to avoid excessive bending of the specimen during the impact. Brass is significantly softer than the RR1000 alloy and hence surface damage due to the support is negligible. However, in the higher impact velocity tests carried out significant plastic deformation of the brass bar is observed. Therefore, it is essential that the FE model include this plastic deformation, as it is a relevant source of energy dissipation during the impact tests.

Symmetry of the problem was exploited and only $1 / 4$ of the rig was modelled. The FE model geometry and boundary conditions are given in Fig. 5. The compliance of the rig support was modelled by springs. The elastic stiffness of these springs significantly change the final geometry of the dent and the rebound velocity. Calibration of the compliance was carried out by comparing the model with the experimental rebound velocities measured by high speed camera. An elastic-plastic material model for the Nickel superalloy was provided by our industrial collaborator and it was implemented as LS-DYNA piecewise linear elastic-plastic Material $24^{1}$, where the Young's modulus and Poisson ratio are given by $E=220 \mathrm{GPa}$ and $v=0.24$ (Preuss et al., 2006). The hardened steel indenter tip was assumed to be purely elastic (LS-DYNA Material 1 ). The brass bar used in the experiments to reduce the flexibility of the Nickel specimen was also modelled and a simple elastic perfectly plastic material model was used to capture the plastic deformation on the brass bar at higher impact velocities. The material properties used for the brass block are: $E=97 \mathrm{GPa}, v=0.31, \sigma_{Y}=150 \mathrm{MPa}$. Linear hexagonal elements with reduced integration were used in the analysis

\footnotetext{
${ }^{1}$ Due to the confidentiality agreement between the University of Oxford and Rolls-Royce plc we are unable to provide the exact elastic-plastic material data of the RR1000 alloy used in this analysis.
} 
and hourglass stiffness was added to the elements to avoid excessive deformation of the elements near the notch root. The hourglass stiffness adds artificial energy to the system but this was monitored and assured to be less than $10 \%$ of the total energy. A convergence analysis was carried out and several mesh sizes were compared. The final mesh size chosen has a element size near the centre of the notch of $h_{e}=0.05 \mathrm{~mm}$.

\subsection{Calibration of the FE model}

The only parameter that requires calibration in the FE model is the stiffness of the support springs, $k$, in Fig. 5. An appropriate way of choosing the stiffness is so as to produce the best fit between the numerical and experimental rebound velocities. A number of FE simulations with arbitrary values of $k$ were carried out for the midpoint in the velocity range tested $(6.221 \mathrm{~m} / \mathrm{s})$ and the rebound velocity was obtained as a function of the spring stiffness. The value of the stiffness corresponding to the experimentally measured rebound velocity was obtained by interpolation and this gave an optimum stiffness value of $k=3 \times 10^{3} \mathrm{~N} / \mathrm{mm}$. This value of $k$ results in a similar elastic response of the model as that given by the rig during the tests. We neglect here any dissipation that may occur, i.e. no damping is added to the model.

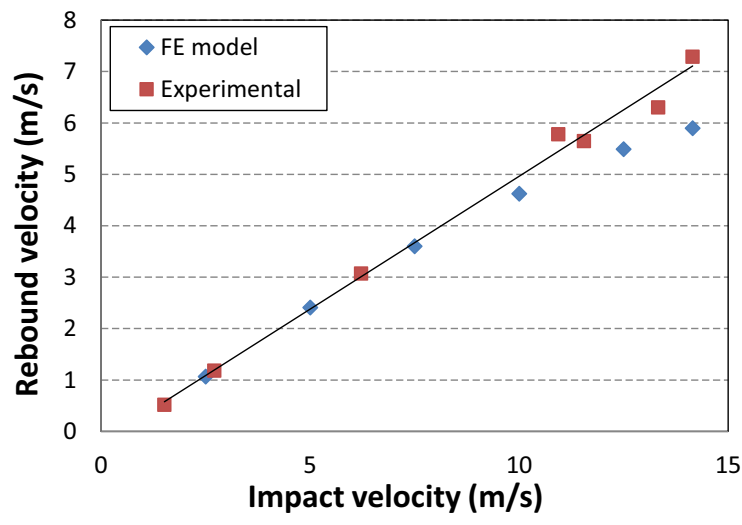

Figure 6: Rebound velocity as a function of the impact velocity.

The comparison between the experimental and the FE relationships between impact velocity and rebound velocity is presented in Fig. 6. The quality of the FE results when the spring stiffness is adjusted appropriately is very good for most of the velocity range. At higher impact velocities there is a small discrepancy between the FE and experimental results. This can be explained by the increased plastic deformation of the brass bar support and the relatively simple material model used. However, this difference does not significantly affect the prediction of the dent depth, as will be shown later. Note that the calibration spring stiffness and only needs to be carried out at a single velocity. We will then assume that once the rebound speed of the FE model is correct, the energy dissipated during the impact of the indenter is the same as that observed experimentally. Hence, we should expect similar plastic deformations and similar geometries of the dents in the FE results and as measured experimentally with the Alicona microscope.

\subsection{Dent geometry validation}

The geometry of the dents obtained in the explicit FE model is now compared with experiment. First, an implicit analysis was carried out at the end of the explicit analysis to eliminate all the dynamic effects after the impact. since no damping was added to the system in the FE model and hence stress waves will permanently travel back and forth. These are eliminated from the final solution by performing a "springback" analysis at the end of the explicit dynamic simulation. All the information regarding stresses and strains of each element are stored in the "dynain" file and a static analysis is carried out to obtain the equilibrium solution. The geometry of the dents obtained numerically were measured at the end of this static equilibrium step. These are then compared with the geometry of the dents obtained experimentally. The FE and experimental relationships between dent depth and impact velocity are presented in Fig. 7a. Good agreement between the two sets of results is observed. Although the error of the FE solution seems to increase at higher impact velocities, the relative error of the FE results with respect to the experimental data at the highest impact velocity analysed, $14.15 \mathrm{~m} / \mathrm{s}$, remains below $15 \%$. Note that one experimental point lies outside the 
general trend, but this is the test referred to above which had a double impact and, hence, the depth of the dent is greater than for a single impact of the indenter. Good agreement between the FE results and experimental data for the length and width of the dents were also observed, as displayed in Fig. $7 \mathrm{~b}$.

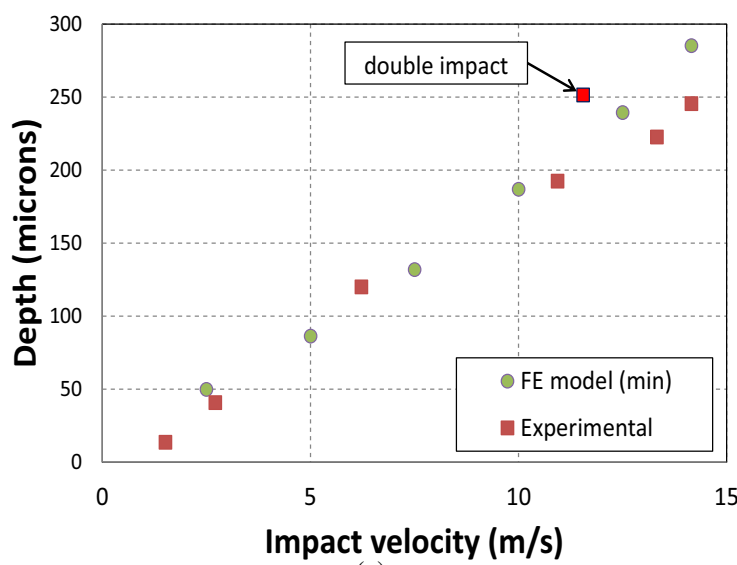

(a)

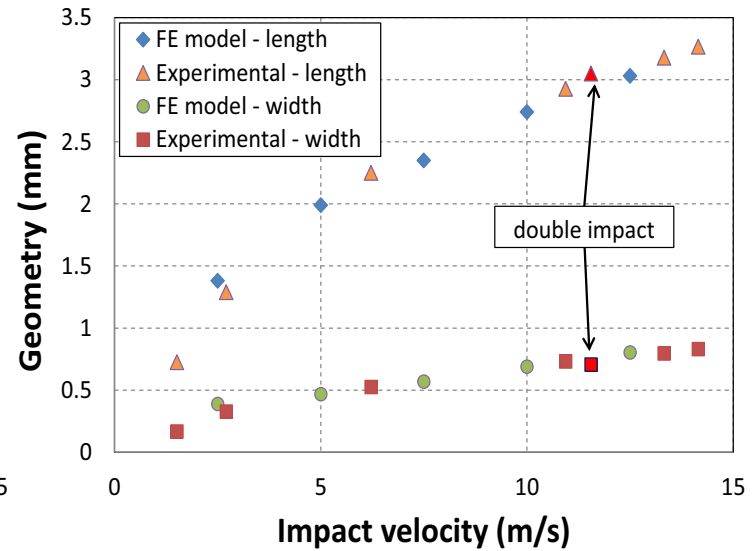

(b)

Figure 7: Comparison between FE and experimental maximum (a) depth and (b) length and width of the dents as a function of the impact velocities.

\subsection{Residual stress distribution}

An example of residual stress field obtained with the FE model after the strike at an impact velocity of $10 \mathrm{~m} / \mathrm{s}$, and causing a dent with a depth of approximately $200 \mu \mathrm{m}$, is shown in Fig. 8. Note that under the centre of the stress, the component $\sigma_{x x}$ has the largest range, with stresses varying from the high tensile stress near the root to the subsequent high compressive stress zone, although large compressive direct stresses in the $z$-direction may also be observed under the dent root. The stress in the $y$-direction, however, is negligible compared with the other two components of direct stress. The stress component displayed in Fig. 8a is the stress along the axial direction of the specimen (here taken as the $x$-axis). This is the direction in which the fatigue loads will later be applied and hence is the most relevant component of stress for crack propagation. Note that a significant compressive zone is created under the dent. This compressive zone will be likely to retard crack propagation. On the other hand, the dent acts as a geometric stress concentrator which will accelerate crack growth. These two competing aspects of the damage created need to be taken into account when evaluating the impact of the damage on the fatigue life of components.

The predicted residual stress component $\sigma_{x x}$ under the deepest point of the dent is presented in Fig. 9. For all velocities the stress distribution is tensile near the base of the dent, due to elastic recovery after the impact. Then, a significant sized compressive zone may be observed. The main difference between the different impact velocities is the extent of the compressive zone; the higher the impact velocity the bigger the compressive zone. The residual stress distribution will have a significant contribution to the propagation of cracks during the fatigue test. Despite the good agreement between the calibrated FE model and the experimental dent geometry, it is important that the residual stresses generated around the dent are also validated by comparing with experimentally measured stresses.

\section{Residual stress measurement}

\subsection{Methodology and calibration}

The diffraction analysis was carried out in a laboratory source-based X-ray diffractometer (Bruker D8) at the HEX lab, Oxford. The stresses were measured by the $\sin ^{2} \psi$ method (Prevéy, 1986). First, a calibration test with the R1000 superalloy was carried out to obtain reflection-specific X-ray elastic constants for this alloy and to validate the implementation of the $\sin ^{2} \psi$ residual stress measurement technique. Note that Nickel crystals have orthotropic elastic properties and each lattice direction has different elastic stiffness, which depend on the crystal structure (FCC for Nickel alloys) and on the anisotropy, or Zener's factor, of the alloy. A tension-compression uniaxial rig was 


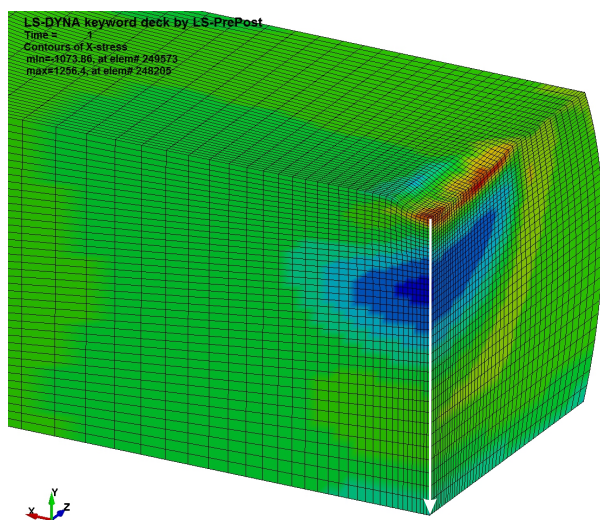

(a)
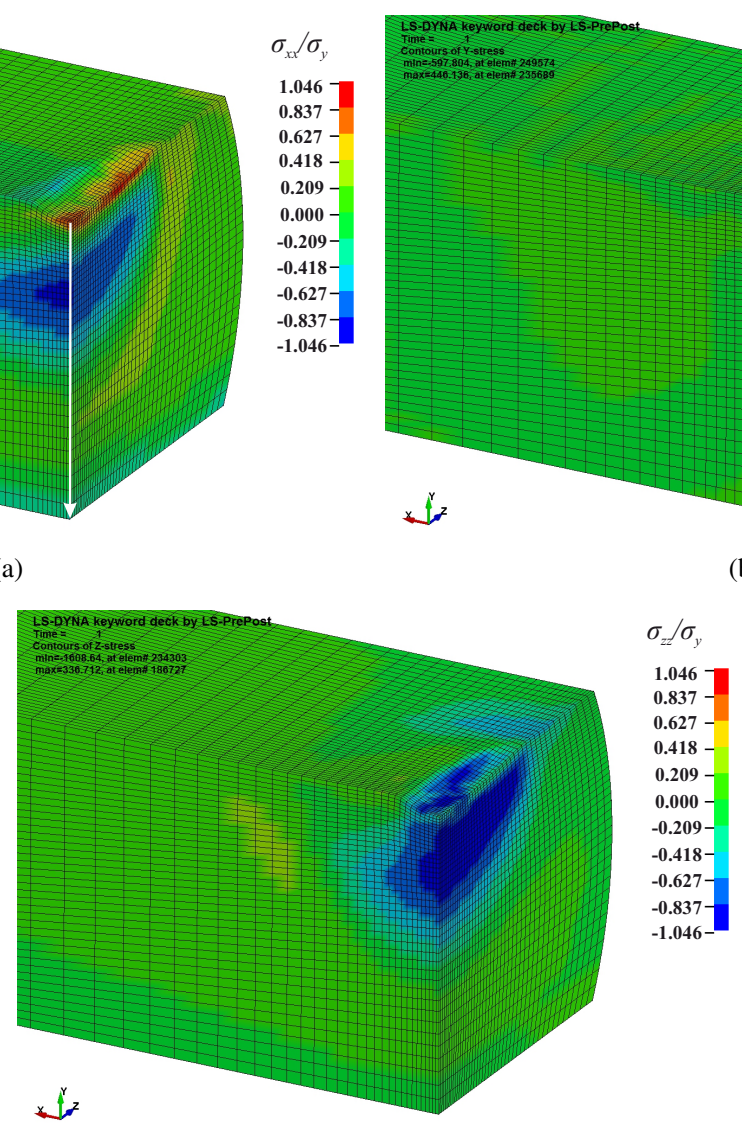

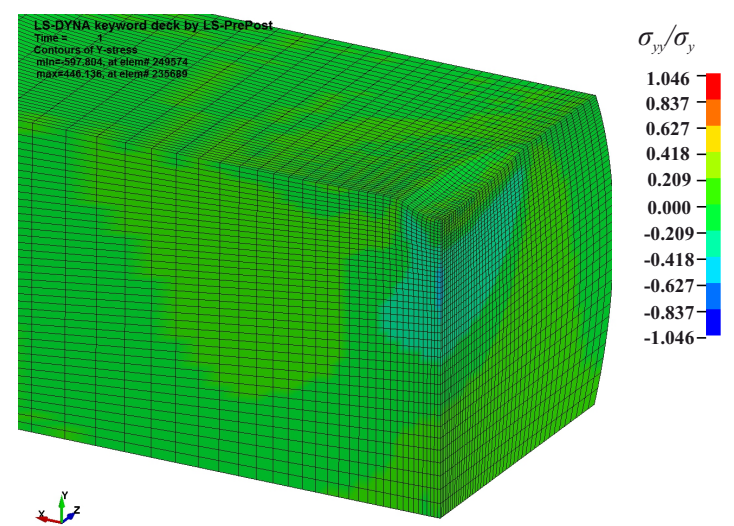

(b)

(c)

Figure 8: Contour plot of residual stress component (a) $\sigma_{x x}$; (b) $\sigma_{y y}$ and (c) $\sigma_{z z}$ obtained from the FE model for an impact velocity of $10 \mathrm{~m} / \mathrm{s}$.

mounted on the diffractometer to control the stress applied to a calibration sample. A "dog-bone" shaped sample, made of similar RR1000 superalloy, was wire-cut and prepared by successive fine grinding and polishing with a $3 \mu \mathrm{m}$ water based monocrystalline diamond suspension. The sample had a cross-section of $1.97 \mathrm{~mm}^{2}(2.01 \times 0.98 \mathrm{~mm})$ and a maximum load of $900 \mathrm{~N}$ was applied, resulting in a stress at the centre of the specimen of approximately $450 \mathrm{MPa}$. For the calibration of the X-ray elastic constants, the sample was loaded to four different load levels: $10 \mathrm{~N}, 310 \mathrm{~N}, 610 \mathrm{~N}$ and $910 \mathrm{~N}$. First, a force of $10 \mathrm{~N}$ was applied to the sample and the stresses measured. Then, the sample was loaded to $910 \mathrm{~N}$ and reduced to $610 \mathrm{~N}$ and $310 \mathrm{~N}$, respectively. The sample was unloaded back to $10 \mathrm{~N}$ and the stress measurement repeated. For each load level the diffraction pattern was obtained for five $\psi$ angles $\left(0^{\circ}, 20.70^{\circ}, 30.00^{\circ}, 37.76^{\circ}, 45.00^{\circ}\right)$, resulting in five equally spaced $\sin ^{2} \psi$ points $(0,0.125,0.25,0.375,0.5)$. The X-ray source was set to $40 \mathrm{kV}$ and $30 \mathrm{~mA}$ and at each angle $\psi$ the sample was exposed for $300 \mathrm{~s}$. The sample was positioned at an angle $\omega=42^{\circ}$ with respect to the source and the angle of the 2D detector (Vantec) was set as $2 \theta=84^{\circ}$, such that peaks corresponding to the $\langle 200\rangle,\langle 311\rangle$ and $\langle 220\rangle$ directions were captured using copper source with wavelength of $1.5406 \AA$.

\subsection{Residual stress calculation}

The output of the XRD measurements are the diffraction peaks patterns. In this analysis these are the peaks associated with crystal directions $\langle 200\rangle,\langle 311\rangle$ and $\langle 220\rangle$. The residual stress is measured from the change in the peak location, which is related to the change in lattice spacing of crystal planes by the Bragg's Law,

$$
d=\frac{\lambda}{2 \sin \theta},
$$




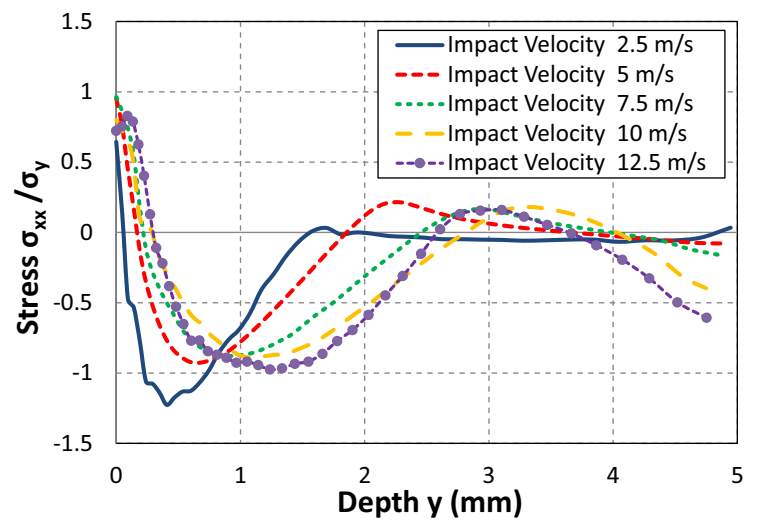

Figure 9: FE results of the $\sigma_{x x}$ residual stress distribution under the notch root for different impact velocities.

where for the copper sources used, the wavelength is $\lambda=1.5406 \AA$.

In order to find the location, $2 \theta$, of the centre of each diffraction peak the experimental diffraction data was processed in MATLAB and curve fitting using the Lorentzian function was carried out, where one of the fitting parameters gives the position of the peak centre. The peak centre shift is presented in Appendix A for the calibration tests carried out with the "dog-bone" specimen and the in situ tensile Deben rig. The lattice spacing, $d$, is obtained from Eq. (1) and plotted as a function of $\sin ^{2} \psi$. The slope, $m$, and the intersection with the vertical axis, $d_{\phi 0}$ (i.e. the spacing for $\psi=0$ ), of the curve correlating the lattice spacing and the $\sin ^{2} \psi$ values can be obtained. Prevéy (1986) has shown that the unloaded lattice space $d_{0}$ varies from $d_{\phi 0}$ by not more than $\pm 1 \%$, and that a good approximation of the residual stresses may be obtained with the approximation $d_{\phi 0} \simeq d_{0}$. The stresses were calculated by using Prevéy's approximation and are given by

$$
\sigma_{\phi}=\left(\frac{E}{1+v}\right)_{\langle h k l\rangle} \frac{m}{d_{\phi 0}} .
$$

Note that Eq. (2) uses the X-ray elastic constants for each crystallographic direction $\langle h k l\rangle$ normal to the lattice planes in which the strain are measured. However, the stresses may also be calculated by using the bulk Young's modulus, $E$, if the average of the three peaks is used. Korsunsky (2008) has shown that the average of the stresses calculated for multiple peaks with the bulk elastic parameters gives a good estimate of the stress. The plot of applied stress versus measured stress using the bulk elastic parameters approximation is shown in Fig. 10a. Note that although the curve for each peak has a different slope, the slope of the average is very similar to the bulk response, i.e. the slope is approximately 1 . The X-ray elastic constants of the RR1000 alloy were also obtained for the three peaks analysed. They are calculated from the slope, $m^{*}$, of the curves given by the lattice space variation, $\Delta d$, as a function of the applied stress (Fig. 10b). The X-ray elastic constants may be calculated by the following expression (Prevéy, 1977),

$$
\frac{E_{\langle h k l\rangle}}{\left(1+v_{\langle h k l\rangle}\right)}=m^{*} d_{0\langle h k l\rangle} \sin ^{2} \psi
$$

where $E_{\langle h k l\rangle}$ is the X-ray elastic constant for each $\langle h k l\rangle$ peak. The X-ray elastic constants obtained for each of the three peaks analysed are presented in Table 3. The ratio between the X-ray elastic constants between the peaks and a principal direction of the crystal, e.g. the $\langle 100\rangle$ direction, is also given. For comparison, we also present the elastic stiffness ratio for the same crystallographic directions of a known single-crystal alloy and the relative difference between the ratios. A small difference in ratio between the $\langle 311\rangle$ and $\langle 100\rangle$ is observed between the RR1000 sample and the single-crystal CMSX-4 alloy. However, the stresses will be measured by the averaging of the stresses with the bulk elastic parameters approach and the X-ray elastic constant approach will be used only to give us a measure of the experimental error associated with the measurements. 
Table 3: RR1000 X-ray elastic constants.

\begin{tabular}{ccccc}
\hline $\begin{array}{c}\text { Lattice } \\
\text { plane }\end{array}$ & $\begin{array}{c}\mathbf{E}_{\langle\mathbf{h k l}\rangle} \\
(\mathbf{G P a})\end{array}$ & $\begin{array}{c}\text { Measured } \\
\mathbf{E}_{\langle\mathbf{h k l}\rangle} / \mathbf{E}_{\langle\mathbf{1 0 0}\rangle}\end{array}$ & $\begin{array}{c}\text { CMSX-4 } \\
\mathbf{E}_{\langle\mathbf{h k l}\rangle} / \mathbf{E}_{\langle\mathbf{1 0 0}\rangle}\end{array}$ & $\begin{array}{c}\text { Ratio rel. } \\
\text { error }(\boldsymbol{\%})\end{array}$ \\
\hline$\langle 311\rangle$ & 197.8 & 1.091 & 1.387 & 20.31 \\
$\langle 220\rangle$ & 314.9 & 1.737 & 1.799 & 3.46 \\
$\langle 200\rangle$ & 181.3 & 1 & 1 & 0 \\
\hline
\end{tabular}

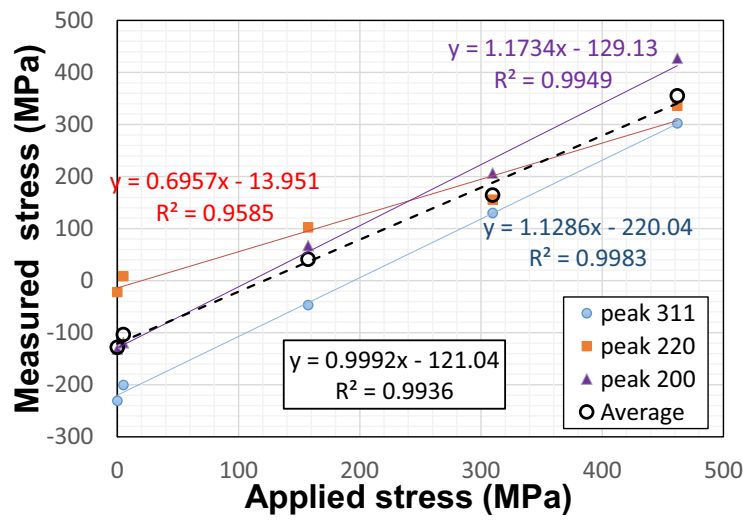

(a)

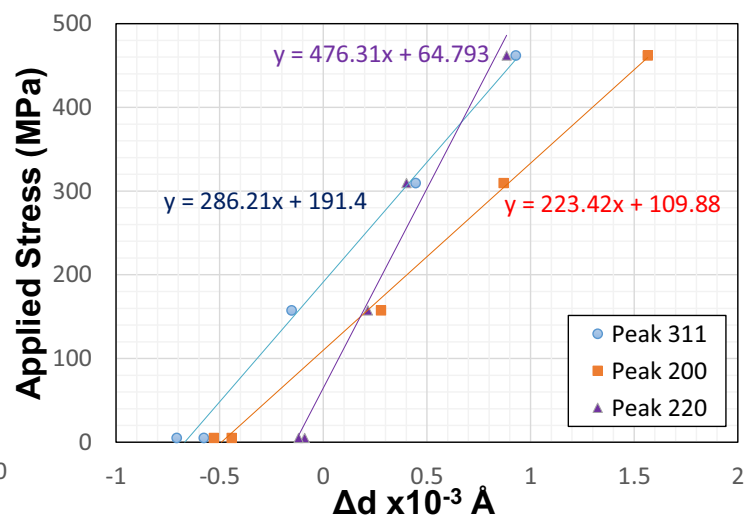

(b)

Figure 10: (a) Applied stress versus measured stress for the three peaks and average using the bulk Young's modulus approximation; (b) Variation of the lattice spacing $\Delta d$ as a function of the applied stress.

\subsection{Measurement of dented specimens}

The same procedure used in the calibration analysis was then repeated on the dented samples. Four specimens were selected for residual stress measurement (in Table 2, Tests 1, 3, 6 and 8). These specimens have depths at the centre of the dent ranging from $40-245 \mu \mathrm{m}$. The specimens were sectioned at the centre of the dent transverse to the longest direction (Fig. 3). The sectioning of the specimens was carried out with a diamond disc saw at low rotation speed and applied force, in an attempt to preserve as best as possible the residual stresses under the dent. Once sectioned, the samples were carefully polished down to $3 \mu \mathrm{m} R_{a}$. The $\sin ^{2} \psi$ technique was then applied on the dented samples, where the diffraction pattern was also obtained for the five tilt angles $\psi\left(0^{\circ}, 20.70^{\circ}, 30.00^{\circ}, 37.76^{\circ}, 45.00^{\circ}\right)$. Measurements under the dent were made at several points $y_{i}$ away from the base of the dent, $y_{0}$ (Fig. 11). The $\sigma_{x x}$ component of stress was measured and the rotation $\psi$ of the sample during the $\sin ^{2} \psi$ method for residual stress measurement was carried out as illustrated in Fig. 11.

The experimental measurements were carried out with both the bulk elastic parameters and the X-ray elastic constants approaches. The residual stress distribution measured under the dent root of sample SD011 $(d=246 \mu \mathrm{m})$ are displayed in Fig. 12. As expected, the results show a high tensile stress near the dent root and a decay in stress as the observation point moves away from it. Note that the coordinate $y / d_{\text {depth }}$ in Fig. 12 is the coordinate of the

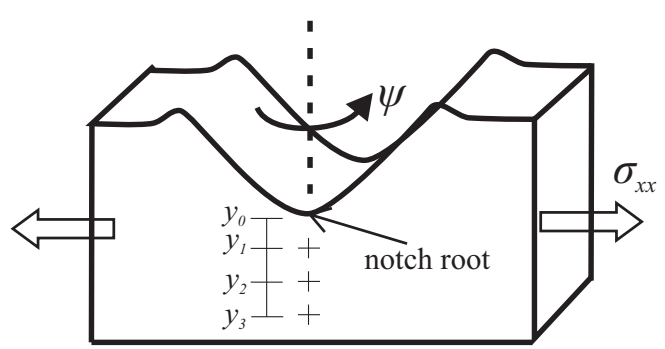

Figure 11: Schematic representation of dented sample; measurement points under the dent root, tilt angle $\psi$ and direction of loading. 


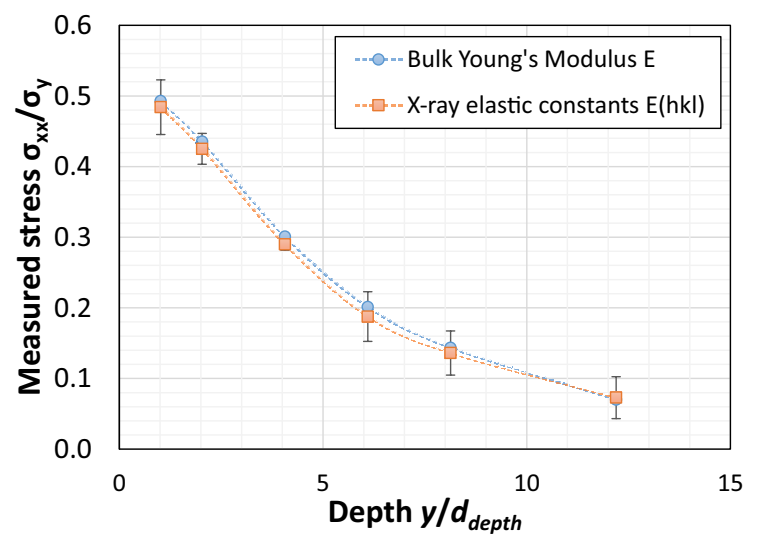

Figure 12: Measured residual stress as a function of the distance to the notch root; test number $8($ depth $=246 \mu \mathrm{m})$.

centre of the beam, and some of the sampling volume may be outside the specimen for the points close to the dent root. It is important to highlight that the measured stress is in fact an average over the area of the projection of the $\mathrm{X}$-ray beam size onto the specimen. The beam spot size was measured in situ in the Bruker D8 diffractometer with an $\mathrm{X}$-ray Eye camera (Fig. 13). The beam spot is approximately $2 \mathrm{~mm}$ in diameter. The beam intensity has a Gaussian distribution with the full-width-half-maximum (FWHM) of 1.6 in the horizontal direction and a bimodal Gaussian distribution in the vertical direction. In interpreting the results, we should also recognise that some relaxation of the residual stress occurs during sectioning of the sample due to Poisson effect during the sectioning of the specimen. This may explain why a compressive stress region is not observed in the XRD measurements. We use the deviation of the stresses measured, using the X-ray elastic constant approach, between the different diffraction peaks as an estimation of the error associated with the experimental measurements. These are displayed in the form of error bars in Fig. 12. Similar results were obtained for the other three dented samples. The residual stresses measured for all four samples are shown in the next section, together with the FE model results.

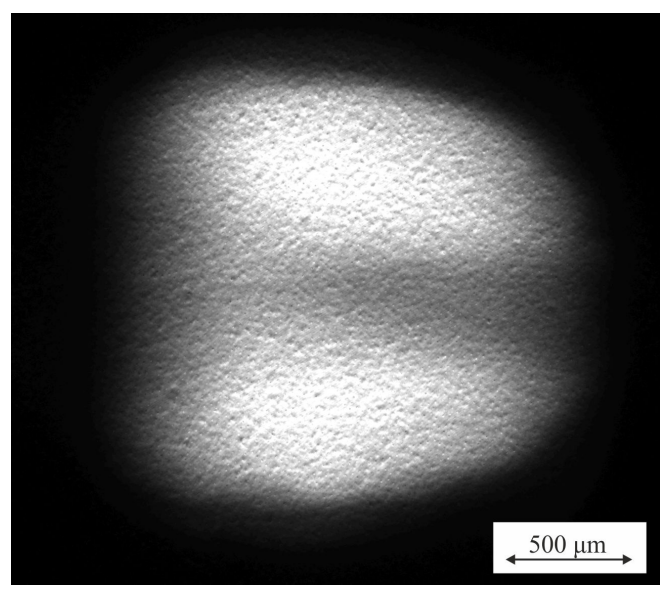

Figure 13: X-ray beam spot size measured with an in-situ X-ray Eye camera

\section{Comparison between XRD and FE results}

The FE results of stress distribution under the dent in Fig. 9 may not be directly compared with the experimentally measured residual stresses since the effect of material removal and stress averaging over the size of the X-ray beam needs to be taken into account. First, the area and weight function for the stress averaging needs to be defined. This 
has to be similar to the averaging of stresses in the projected area of the X-ray beam during the XRD measurements. Using the information obtained with the X-ray Eye camera, a beam size of radius of approximately $r=1 \mathrm{~mm}$ chosen. Note that the sample is positioned at an angle $\omega=42^{\circ}$ with respect to the source, such that the beam area project on the inclined plane is given by an ellipse with the large and small radii given by $r / \sin \omega$ and $r$, respectively. A Gauss weighting function was applied to average the stresses inside the ellipse such that the FWHM $=1.6$ and the distance between the bimodal peaks in the vertical direction is $1 \mathrm{~mm}$.

The material removal during the sectioning of the samples also need to be taken into account in the FE results before comparing them with the XRD measurements. This is done by removing the symmetry boundary conditions applied to the $x-y$ plane during the static equilibrium step (or "springback" analysis). The distance of the measured plane, i.e. the face of the samples, to the centre of the original dent mark was measured and recorded during the sectioning and polishing of the samples. The correct distance from the centre of the dent to actual plane measured after polishing was obtained in the FE model by removing elements in the $z$ direction up to the distance to the dent centre recorded for each sample. The precision of the "material removal" in the FE model is limited by the discretisation of the specimen in the model, i.e. the element size. The final contour plot result of the stress component $\sigma_{x x}$ obtained via the FE model for impact velocities equivalent to the four samples measured are shown in Fig. 14. Note that, once the constraints in the $x-y$ plane are removed, the region near the face of the specimen normal to the $z$ direction has no longer a high compressive zone as it is observed in Fig. 9. This is due to the fact that the specimens are now free to deform in the $z$ direction, relaxing some of the existing residual stress $\sigma_{x x}$ due to Poisson effect.

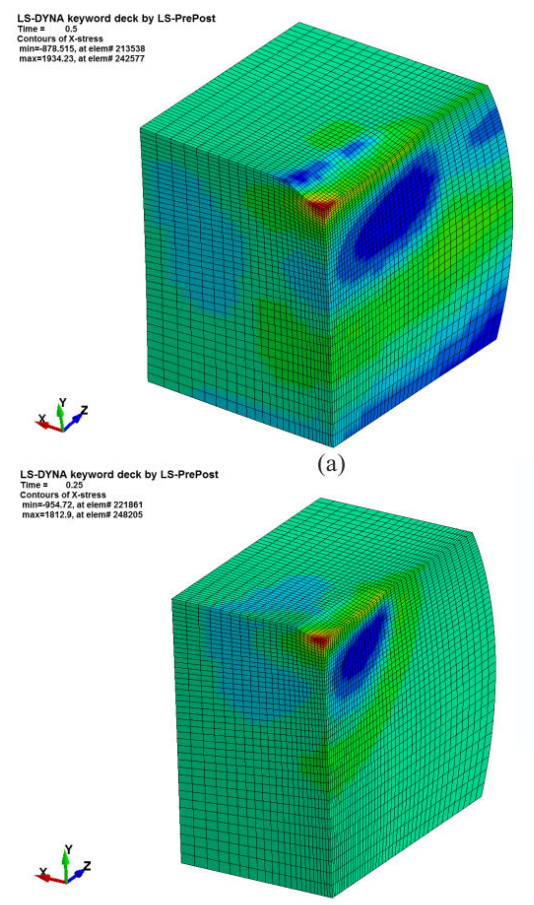

(c)

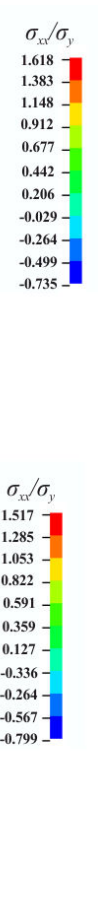

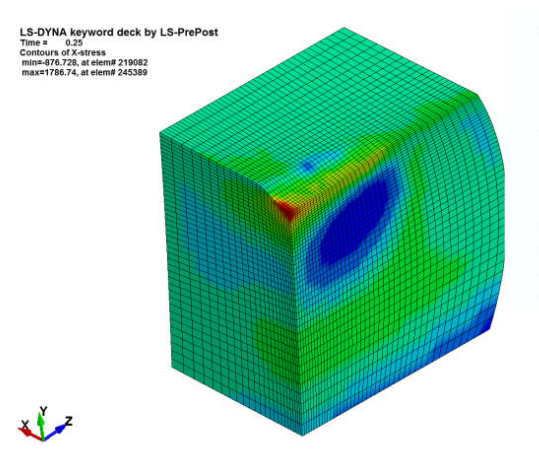

(b)

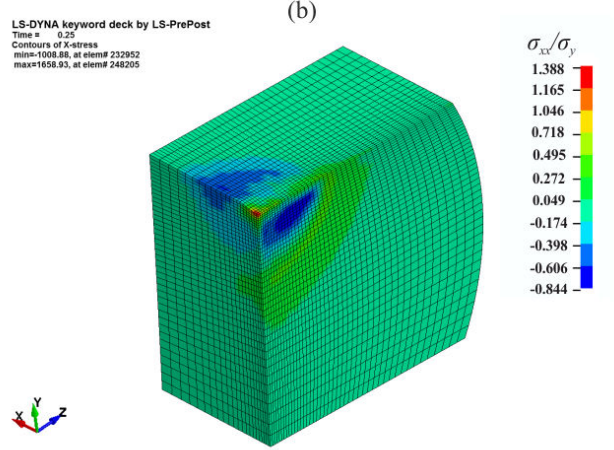

(d)

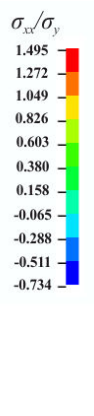

$\sigma_{x x} / \sigma$

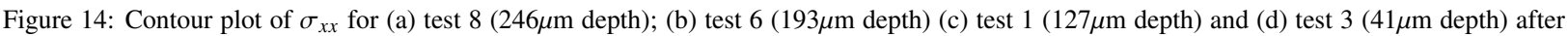
"sectioning" in the FE model.

Once the averaging procedure of stress over the projected X-ray beam size area on the sample and the effects of the material removal was implemented, a comparison between FE results and XRD measurements was carried out. The results are shown in Fig. 15. The red dots represent the experimental results, and include error bars as described in Section 4.3. The blue lines are the averaged FE results using the numerical procedure outlined above. Very good agreement between FE results and XRD measurements near the dent root is observed for tests number 8, 6 and 1 . The stress decay as the observation point moves away from the notch root is also consistent between both numerical and experimental results. 


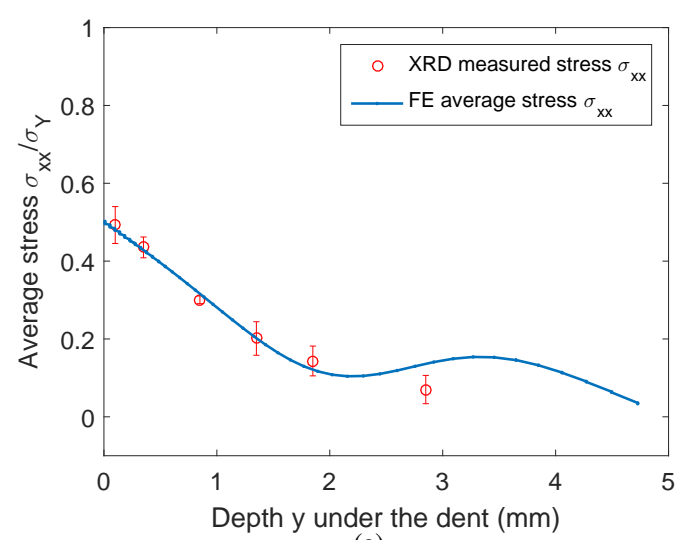

(a)

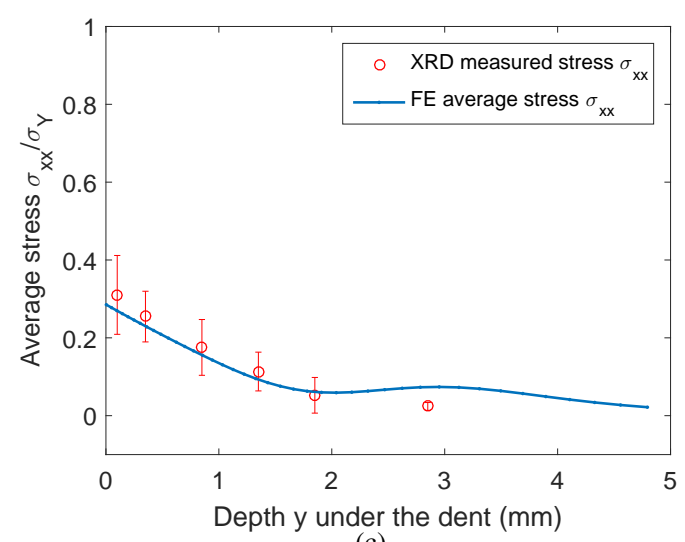

(c)

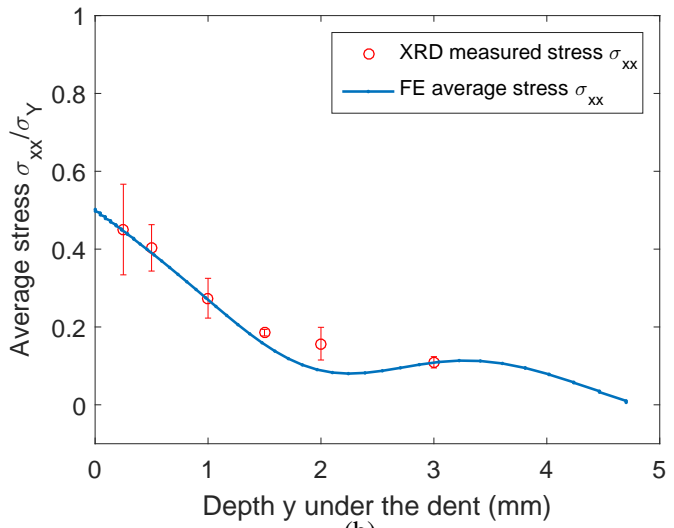

(b)

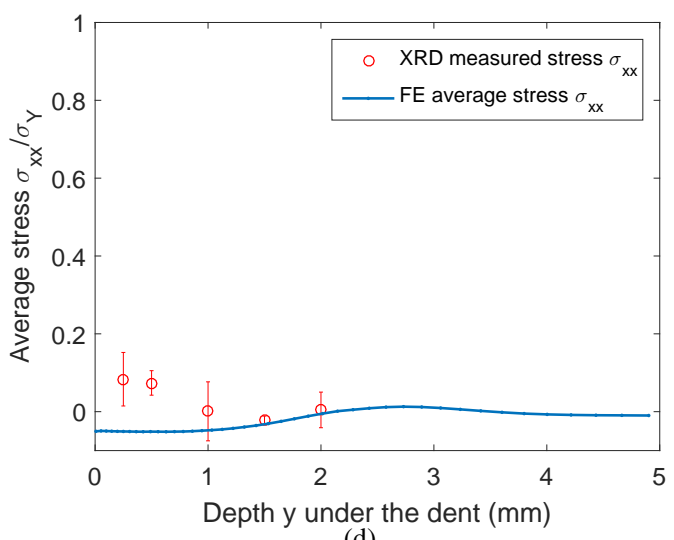

(d)

Figure 15: Finite element stress profile (blue dot) and XRD measured stress (red circles) of: (a) test 8 (246 $\mu \mathrm{m}$ depth); (b) test 6 (196 $\mu \mathrm{m}$ depth); (c) test $1(127 \mu \mathrm{m}$ depth); (d) test $3(41 \mu \mathrm{m}$ depth).

Test number 3, however, has a difference of about $180 \mathrm{MPa}$ at the root of the dent between the FE model results and the XRD measurements. The size of the dent of test number 3 sample is very small, and so is the magnitude of the residual stress. This makes the sample more sensitive to experimental errors, which could be due to issues such as surface preparation and alignment. The error between the FE model and the experimental measurements near the dent root of test number 3 is consistent with those caused by surface grinding and polishing which can reach up to $200 \mathrm{MPa}$ (Duó et al., 2007). Improvements might be achieved with a more careful surface preparation, using colloidal silica polishing or even electro-polishing.

\section{Discussion}

The explicit dynamic FE model presented in this paper to simulate handling and service damage gives accurate prediction of dent geometry and residual stresses in its vicinity. The calibration of the model was obtained by choosing an optimum stiffness of the springs. The springs in the model emulate the experimental rig compliance and, once calibrated, a similar rebound velocities to those observed with the high speed camera is obtained with the FE model. This calibration is essential for the accuracy of the results, since it assures that the net input of energy into the system during impact is matched between the FE model and the impact test, i.e. the difference in kinetic energy due to the impact and rebound velocities of the striker. Part of the energy input into the system is transformed in elastic energy of the rig, which in the tests are quickly dissipated, but in the FE model produces a constant oscillation of the springs due to the absence of damping. The equilibrium solution is obtained by carrying out a static implicit analysis at the 
end of the dynamic analysis to eliminate the transient oscillations from the final dent geometry and residual stress field. The calibration of the spring stiffness also controls how much energy is transferred to the specimen and how much is "dissipated" in the form of kinetic energy of the rig in the FE model.

The good agreement between the FE model and the experimental results relies on the accuracy of the elastic-plastic material data used. The data was assumed to be valid under dynamic conditions. Strain-rate sensitivity of alloys can have significant impact on the depth of the dent and residual stresses (Chen and Hutchinson, 2002). However, unlike Titanium alloys, which are the main focus of FOD analyses, Nickel superalloys are less sensitive to dynamic effects, such as strain-rate hardening (Cornet et al., 2014; Preuss et al., 2006). Even if the impact velocity is relatively low when compared with FOD, the strain rate in these tests may reach the order of $10^{3} s^{-1}$. Hence, a higher discrepancy between the FE and experimental results would be expected if the material analysed were more strain-rate sensitive. The lack of dynamic sensitivity of the material data used in the analysis poses the question: why wasn't a static model enough to characterise the residual stress field near the dent? Chen and Hutchinson (2002) have shown that accounting for inertia is important to predict the depth and size of the dent created. However, in the absence of rate-dependent material parameters, the residual stress generated from impact or from indentation will be identical, provided the depth and geometry of the dent are the same. This approach has been attempted by Doremus et al. (2015) and it is corroborated by the findings of Boyce et al. (2001). The choice of using a dynamic explicit model, however, is required if one wishes to predict the final geometry and depth of the dent as a function of the impact velocity of the indenter, as well as the residual stress state.

The residual stress predicted by the FE model shows that a significant compressive zone is created under the dent mark. However, a high tensile zone exists near the dent root, which is likely to be relaxed by the existence of micro-cracks near the surface. Although the presence of mircro-cracks appears to have a negligible effect on of the residual stress distribution near the dent, locally near the dent root these will play an important role in relaxing the high tensile zone. The initiation of cracks near the dent root is likely to be accelerated, as found by Doremus et al. (2015), who saw a reduction in initiation time after a thermal treatment was applied to the specimens for the relaxation of the tensile residual stresses near the dent root. Although crack initiation might be accelerated near the notch root, propagation will be significantly affected by the existence of the compressive zone. Under some conditions of loading, cracks will initiate but the stresses may not be enough to break through the compressive zone. However, before any conclusions can be drawn regarding the impact of the residual stress field surrounding the dent on the fatigue life of components, further investigation is required, particularly concerning the relaxation of the residual stresses due to mechanical loading and/or thermal expansion at high temperatures (Boyce et al., 2003).

\section{Conclusions}

A characterisation study of dents due to the low speed impact of a hard indenter on the RR1000 Nickel superalloy was presented in this paper. Dents were created on the specimens by laboratory impact tests on a modified Hopkinson Bar rig. The dent geometry and depth were obtained as a function of the impact velocity and used to validate, geometrically, the FE model presented. The model was calibrated by adjusting the stiffness of the fixture support in the model so as to match the measured rebound velocity of the indenter. Good predictions of dent geometry were then obtained.

Validation of the residual stress field obtained numerically was carried out by comparing the FE results with stresses measured using laboratory XRD. It was shown that by taking into account the material removal, which relaxes the residual stresses, and averaging over the X-ray beam spot size, the FE results compare well with experiment. The results are more accurate for the deepest dents analysed. The shallowest dent shows the biggest difference between experiment and prediction. However, this has the lowest stress levels, and results will be more sensitive to surface preparation and alignment of that sample. Improvements in the XRD procedure and more careful sample preparation may reduce the difference observed. Numerically, the results for low impact velocities have larger elements as a proportion of dent depth. Further analysis with a dent depth dependent mesh, i.e. same ratio of elements per dent depth, might be considered in order to obtain more accurate predictions for shallow dents.

The numerical model presented gives accurate results of stress and dent geometry in the impact velocity range analysed. The stress distribution near the dent can now be used to evaluate the impact of handling and service surface damage on the fatigue life of components. However, careful consideration of on the potential mechanisms of residual stress relaxation needs to be undertaken, before the calculated stress can be used in crack propagation and fatigue life 

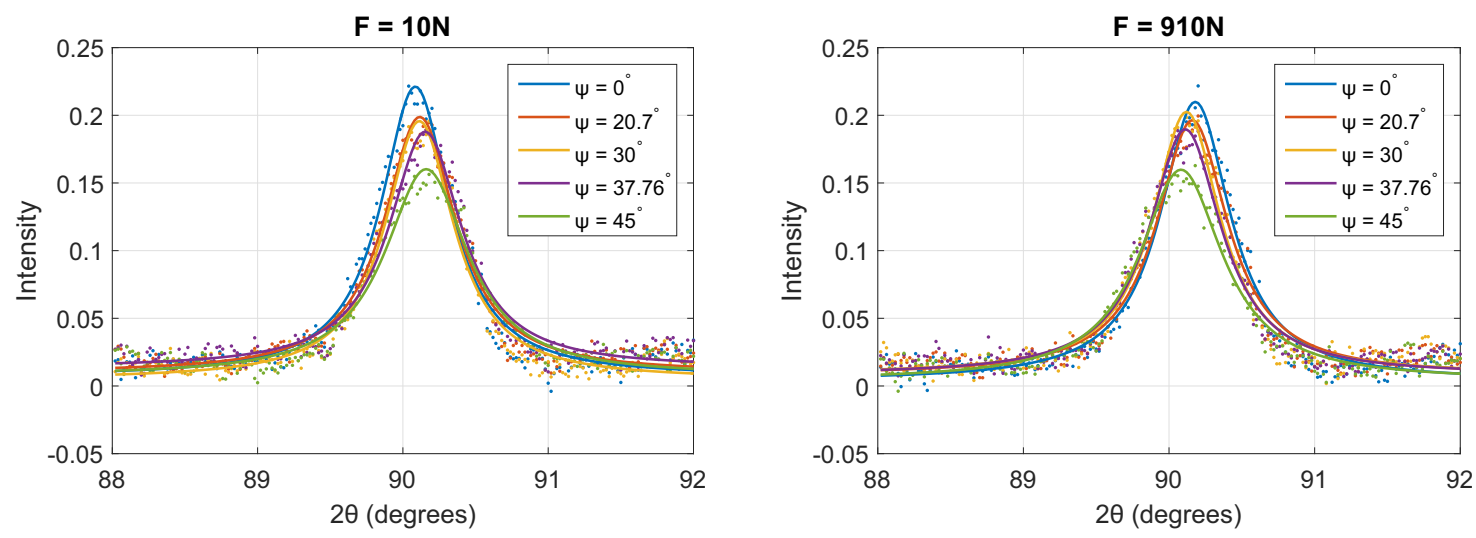

Figure A.16: Measured points and curve fit of the $\langle 311\rangle$ crystal plane for different load levels.

analyses. The FE model and methodology for calibration presented here may also be used for higher impact velocity events, but an improved material model under dynamic conditions and more complex damage models may need to be included.

\section{Acknowledgements}

The authors would like to thank Innovate UK and Rolls-Royce plc for the financial support to this work.

\section{Appendix A. Diffraction pattern for RR1000 alloy}

The diffraction peak $\langle 311\rangle$ obtained during the calibration of the diffractometer rig for the RR1000 alloy are shown in Fig. A.16. The peaks for different applied load $F$ and $\psi$ angle are displayed. The Loretzian function is used in the curve fitting of the experimental points. The stress in the direction of the rotation $\psi$ is given by slope of the location of the centre of the peak for different $\psi$ angles. It is therefore evident that the sample has initially a compressive stress as shown in Fig. 10a, which is probably due to the machining and polishing during the sample preparation. At the highest applied load, $F=910 \mathrm{~N}$, the slope changes direction and the stress becomes positive. The stresses are calculated using the technique described in Section 4.2 and the variation in measured stress between each load level match the applied stress given by the applied force, $F$, over the cross-section area.

\section{References}

Bache, M.R., OHanlon, J., Withers, P.J., Child, D.J., Hardy, M.C., 2016. Characterisation of an advanced nickel based superalloy post cold work by swaging. Metals 6,54 .

Boyce, B., Chen, X., Hutchinson, J., Ritchie, R., 2001. The residual stress state due to a spherical hard-body impact. Mech. Mater. 33, 441-454.

Boyce, B., Chen, X., Peters, J., Hutchinson, J., Ritchie, R., 2003. Mechanical relaxation of localized residual stresses associated with foreign object damage. Mat. Sci. Eng. A 349, 48-58.

Chen, X., Hutchinson, J.W., 2002. Particle impact on metal substrates with application to foreign object damage to aircraft engines. J. Mech. Phys. Solids 50, 2669-2690.

Cornet, C., Wackermann, K., Stöcker, C., Christ, H., Lupton, C., Hardy, M., Tong, J., 2014. Effects of temperature and hold time on dynamic strain aging in a nickel based superalloy. Mater. High Temp. 31, 226-232.

Ding, J., Hall, R., Byrne, J., Tong, J., 2007a. Fatigue crack growth from foreign object damage under combined low and high cycle loading. part i: Experimental studies. Int. J. Fatigue 29, 1339-1349.

Ding, J., Hall, R., Byrne, J., Tong, J., 2007b. Fatigue crack growth from foreign object damage under combined low and high cycle loading. part ii: A two-parameter predictive approach. Int. J. Fatigue 29, 1350-1358.

Doremus, L., Cormier, J., Villechaise, P., Henaff, G., Nadot, Y., Pierret, S., 2015. Influence of residual stresses on the fatigue crack growth from surface anomalies in a nickel-based superalloy. Mat. Sci. Eng. A 644, 234-246.

Duó, P., Liu, J., Dini, D., Golshan, M., Korsunsky, A., 2007. Evaluation and analysis of residual stresses due to foreign object damage. Mech. Mater. 39, 199-211.

Frankel, P., Withers, P., Preuss, M., Wang, H.T., Tong, J., Rugg, D., 2012. Residual stress fields after fod impact on flat and aerofoil-shaped leading edges. Mech. Mater. 55, 130-145. 
Gourdin, S., Cormier, J., Henaff, G., Nadot, Y., Hamon, F., Pierret, S., 2016. Assessment of specific contribution of residual stress generated near surface anomalies in the high temperature fatigue life of a rené 65 superalloy. Fatigue Fract. Eng. M. .

Gourdin, S., Doremus, L., Nadot, Y., Hénaff, G., Pierret, S., 2014. Fatigue crack growth from handling surface anomalies in a nickel based superalloy at high temperature, in: MATEC Web of Conferences, EDP Sciences. p. 16003.

Korsunsky, A.M., 2008. A critical discussion of the $\sin ^{2} \psi$ stress measurement technique, Mater. Sci. Forum, vols. 571-572, pp. $219-224$.

Mall, S., Hamrick, J.L., Nicholas, T., et al., 2001. High cycle fatigue behavior of ti-6al-4v with simulated foreign object damage. Mech. Mater. 33, 679-692.

Mitchell, R., Hardy, M., Preuss, M., Tin, S., 2004. Development of y prime morphology in p/m rotor disc alloys during heat treatment. TMS superalloys 2004, 361-370.

Nowell, D., Duó, P., Stewart, I., 2003. Prediction of fatigue performance in gas turbine blades after foreign object damage. Int. J. Fatigue 25, 963-969.

Peters, J., Boyce, B., Chen, X., McNaney, J., Hutchinson, J., Ritchie, R., 2002. On the application of the kitagawa-takahashi diagram to foreignobject damage and high-cycle fatigue. Eng. Fract. Mech. 69, 1425-1446.

Preuss, M., Withers, P., Baxter, G., 2006. A comparison of inertia friction welds in three nickel base superalloys. Mat. Sci. Eng. A $437,38-45$.

Prevéy, P.S., 1977. A method of determining the elastic properties of alloys in selected crystallographic directions for x-ray diffraction residual stress measurement. Advan. in X-ray Anal. 20, 345-354.

Prevéy, P.S., 1986. X-ray diffraction residual stress techniques. ASM International, ASM Handbook. 10, 380-392. 